\title{
Total Dominating Sets and Total Domination Polynomials of Square Of Wheels
}

\author{
T.Premala, Dr.C.Sekar, \\ Assistant Professor/ Mathematics, C.S.I.Institute of Technology, Thovalai \\ Associate Professor/Mathematics, Aditanar college of Arts and Science,Tiruchendur
}

\begin{abstract}
Let $G=(V, E)$ be a simple connected graph. A set $S \subseteq V$ is a total dominating set of $G$ if every vertex is adjacent to an element of $S$. Let $D_{t}\left(W_{n}^{2}, i\right)$ be the family of all total dominating sets of the graph $W_{n}^{2}$, $n \geq 3$ with cardinality $i$, and let $d_{t}\left(W_{n}^{2}, i\right)=\left|D_{t}\left(W_{n}{ }^{2}, i\right)\right|$. In this paper we compute $d_{t}\left(W_{n}{ }^{2}, i\right)$, and obtain the polynomial $D_{t}\left(W_{n}^{2}, x\right)=\sum_{\mathrm{i}=\mathrm{Y}_{\mathrm{t}}\left(\mathrm{W}_{\mathrm{n}}^{2}\right)} d_{t}\left(W_{n}^{2}, i\right) x^{i}$, which we call total domination polynomial of $W_{n}{ }^{2}, n \geq 3$ and obtain some properties of this polynomial.
\end{abstract}

Keywords: square of wheel, total domination set, total domination polynomial

\section{Introduction}

Let $\mathrm{G}=(\mathrm{V}, \mathrm{E})$ be a simple connected graph. A set $\mathrm{S} \subseteq \mathrm{V}$ is a dominating set of $\mathrm{G}$, if every vertex in $\mathrm{V}-\mathrm{S}$ is adjacent to atleast one vertex in $\mathrm{S}$. A set $\mathrm{S} \subseteq \mathrm{V}$ is total dominating set if every vertex of the graph is adjacent to an element of $\mathrm{S}$. The total domination number of a graph $\mathrm{G}$ is the minimum cardinality of a total dominating set in $\mathrm{G}$, and it is denoted by $\mathrm{Y}_{\mathrm{t}}(\mathrm{G})$. Obviously $\mathrm{Y}(\mathrm{G})<|\mathrm{V}|$. The square of a simple connected graph $\mathrm{G}$ is a graph with same set of vertices of $\mathrm{G}$ and an edge between two vertices if and only if there is a path of length at most 2 between them. It is denoted by $\mathrm{G}^{2}$. We use the notation $\lfloor x\rfloor$ for the largest integer less than or equal to $\mathrm{x}$ and $\lceil\mathrm{x}\rceil$ for the smallest integer greater than or equal to $\mathrm{x}$. Also we denote the set \{ $1,2, \ldots \ldots \ldots \ldots, \mathrm{n}\}$ by $[\mathrm{n}]$, throughout this paper.

In this paper, we study the concept of total dominating sets and total domination polynomials of square of wheels $W_{n}^{2}, n \geq 3$. Let $D_{t}\left(W_{n}^{2}, i\right)$ be the total dominating set of $W_{n}^{2}$ with cardinality $i$. Let $d_{t}\left(W_{n}^{2}, i\right)=\mid D_{t}\left(W_{n}^{2}, i\right)$. The total domination polynomial of $W_{n}^{2}$ is $D_{t}\left(W_{n}^{2}, x\right)=\sum_{i=Y_{t}\left(W_{n}^{2}\right)}^{n+1} d_{t}\left(W_{n}^{2}, i\right) x^{i}$.

\section{Definition 1.1}

The square of a wheel $W_{n}$ is a graph with same set of vertices as $W_{n}$ and an edge between two vertices if and only if there is a path of length atmost 2 between them. It is denoted by $W_{n}^{2}$.

\section{Definition 1.2}

Let $W_{n}^{2}, n \geq 3$ be the square of wheel with $n+1$ vertices. Let $V\left(W_{n}^{2}\right)=\left\{v_{0}, v_{1}, v_{2}, \ldots \ldots v_{n}\right\}$ and $E\left(W_{n}^{2}\right)=$ $\left\{\left(\mathrm{v}_{0}, \mathrm{v}_{\mathrm{i}}\right) ; \mathrm{i}=1,2 \ldots \ldots \ldots, \mathrm{n}\right\} \cup\left\{\left(\mathrm{v}_{\mathrm{i}}, \mathrm{v}_{\mathrm{i}+1}\right) ; \mathrm{i}=1,2, \ldots . \quad \mathrm{n}-1\right\} \quad \mathrm{U}\left\{\left(\mathrm{v}_{\mathrm{i}}, \mathrm{v}_{\mathrm{i}+2}\right) ; \mathrm{i}=1,2, \ldots \ldots \ldots, \mathrm{n}-2\right\} \cup\left(\mathrm{v}_{\mathrm{n}}, \mathrm{v}_{1}\right),\left(\mathrm{v}_{\mathrm{n}-1}, \mathrm{v}_{1}\right)$ ,$\left.\left(v_{n}, v_{2}\right)\right\}$. Let $D_{t}\left(W_{n}^{2}, i\right)$ be the family of total dominating sets of $W_{n}^{2}$ with cardinality $i$ and let $d_{t}\left(W_{n}^{2}, i\right)=1$ $D_{t}\left(W_{n}^{2}, i\right) \mid$. Then the total dominating polynomial $D_{t}\left(W_{n}^{2}, x\right)$ of $W_{n}^{2}$ is defined as $d_{t}\left(W_{n}^{2}, x\right)$ $=\sum_{\mathrm{i}=\mathrm{Y}_{\mathrm{t}}\left(\mathrm{W}_{\mathrm{n}}^{2}\right)}^{\mathrm{n}} \mathrm{d}_{\mathrm{t}}\left(\mathrm{W}_{\mathrm{n}}^{2}, \mathrm{i}\right) \mathrm{x}^{\mathrm{i}}$.

\section{Notation 1.3}

We categorize the total dominating sets of $\mathrm{W}_{\mathrm{n}}^{2}$ into two classes, the total dominating sets containing the vertex $v_{0}$ and the total dominating sets not containing the vertex $v_{0}$, where $v_{0}$ denotes the centre of the wheel.. Let $\mathrm{D}_{\mathrm{t}}^{0}\left(\mathrm{~W}_{\mathrm{n}}^{2}, \mathrm{i}\right)$ be the collection of total dominating sets of $\mathrm{W}_{\mathrm{n}}^{2}$ containing the vertex $\mathrm{v}_{0}$ with cardinality $i$. Let $D_{t}^{1}\left(W_{n}^{2}, i\right)$ be the collection of total dominating sets of $W_{n}^{2}$ not containing the vertex $v_{0}$ with cardinality $i$. The total dominating sets of $W_{n}^{2}$ not containing $v_{0}$ with cardinality $i$ is same as the total dominating sets of the square of cycle $C_{n}^{2}$ with cardinality $i$. Hence $D_{t}^{1}\left(W_{n}^{2}, i\right)=D_{t}\left(W_{n}^{2}, i\right)$.

Let $d_{t}^{0}\left(W_{n}^{2}, i\right)=\left|D_{t}^{0}\left(W_{n}^{2}, i\right)\right|$ and $d_{t}^{1}\left(W_{n}^{2}, i\right)=\left|D_{t}^{1}\left(W_{n}^{2}, i\right)\right|$

So $\mathrm{d}_{\mathrm{t}}^{1}\left(\mathrm{~W}_{\mathrm{n}}^{2}, \mathrm{i}\right)=\mathrm{d}_{\mathrm{t}}\left(\mathrm{C}_{\mathrm{n}}^{2}, \mathrm{i}\right)$

But in general $d_{t}\left(W_{n}^{2}, i\right)=d_{t}^{0}\left(W_{n}^{2}, i\right)+d_{t}^{1}\left(W_{n}^{2}, i\right)$

That is $d_{t}\left(W_{n}^{2}, i\right)=d_{t}^{0}\left(W_{n}^{2}, i\right)+d_{t}\left(C_{n}^{2}, i\right)$

\section{Lemma 1.4}

For $w_{n}^{2}, n \geq 3$ with $\left|V\left(w_{n}^{2}\right)\right|=n+1$, then the total domination number is $Y_{t}\left(w_{n}^{2}\right)=2$ 


\section{Proof:}

In the graph $\mathrm{W}_{\mathrm{n}}^{2}$, a single vertex covers all the remaining vertices of $\mathrm{W}_{\mathrm{n}}^{2}$.

By the definition of total domination, every vertex in total dominating set $\mathrm{S}$ is adjacent to another vertex of $\mathrm{S}$.

Therefore $\mathrm{Y}_{\mathrm{t}}\left(\mathrm{w}_{\mathrm{n}}^{2}\right)=2$.

\section{Remark 1.5}

We have $\mathrm{Y}_{\mathrm{t}}\left(\mathrm{w}_{\mathrm{n}}^{2}\right)=2$

Therefore $\mathrm{D}_{\mathrm{t}}\left(\mathrm{W}_{\mathrm{n}}^{2}, \mathrm{i}\right) \neq \varphi$ if $2 \leq \mathrm{i} \leq \mathrm{n}+1$.

That is $\mathrm{d}_{\mathrm{t}}\left(\mathrm{W}_{\mathrm{n}}^{2}, \mathrm{i}\right) \neq 0$ if $2 \leq \mathrm{i} \leq \mathrm{n}+1$.

\section{Lemma 1.6}

(i) $\quad \mathrm{D}_{\mathrm{t}}\left(\mathrm{W}_{\mathrm{n}}^{2}, \mathrm{i}\right)=\varphi$ if $\mathrm{i}>\mathrm{n}+1$

Let $W_{n}^{2}, n \geq 3$ be the square of wheel with $\left|V\left(W_{n}^{2}\right)\right|=n+1$. Then we have

(ii) $\quad \mathrm{D}_{\mathrm{t}}\left(\mathrm{W}_{\mathrm{n}}^{2}, \mathrm{x}\right)$ is a multiple of $\mathrm{x}^{2}$.

(iii) $\quad \mathrm{D}_{\mathrm{t}}\left(\mathrm{W}_{\mathrm{n}}^{2}, \mathrm{x}\right)$ is a strictly increasing function on $[0, \infty)$.

\section{Proof of (i)}

Since $\mathrm{W}_{\mathrm{n}}^{2}$ has $\mathrm{n}+1$ vertices, there is only one way to choose all these vertices.

Therefore $\mathrm{d}_{\mathrm{t}}\left(\mathrm{W}_{\mathrm{n}}^{2}, \mathrm{n}+1\right)=1$.

Out of these $n+1$ vertices, every combination of $n$ vertices can dominate totally only if $\delta\left(W_{n}^{2}\right)>1$. Therefore $\mathrm{d}_{\mathrm{t}}\left(\mathrm{W}_{\mathrm{n}}^{2}, \mathrm{n}\right)=\mathrm{n}+1$ if $\delta\left(\mathrm{W}_{\mathrm{n}}^{2}\right)>1$.

Therefore $\mathrm{D}_{\mathrm{t}}\left(\mathrm{W}_{\mathrm{n}}^{2}, \mathrm{i}\right)=\varphi$ if $\mathrm{i}\left\langle\mathrm{Y}_{\mathrm{t}}\left(\mathrm{W}_{\mathrm{n}}^{2}\right)\right.$ and $\mathrm{D}_{\mathrm{t}}\left(\mathrm{W}_{\mathrm{n}}^{2}, \mathrm{n}+\mathrm{k}\right)=\varphi, \mathrm{k}=2,3, \ldots \ldots \ldots \ldots$

Thus $d_{t}\left(W_{n}^{2}, i\right)=0$ for $i<Y_{t}\left(W_{n}^{2}\right)$ and $d_{t}\left(W_{n}^{2}, n+i\right)=0$, for $i=2,3$,...

\section{Proof of (ii)}

A single vertex of $W_{n}^{2}$ cannot totally dominate all the vertices of $W_{n}^{2}, n \geq 3$. So the set of all vertices of $W_{n}^{2}$ is totally dominated by atleast two of the vertices of $\mathrm{W}_{\mathrm{n}}^{2}$. Hence the total domination polynomial has no constant term as well as first degree term.

\section{Proof of (iii)}

By the definition of total domination, every vertex of $\mathrm{W}_{\mathrm{n}}^{2}$ is adjacent to an element of total dominating set. That is $D_{t}\left(W_{n}^{2}, x\right)=\sum_{i=Y_{t}}^{n+1}\left(W_{n}^{2}\right)\left|D_{t}\left(W_{n}^{2}, i\right)\right| x^{i}$

Therefore $D_{t}\left(W_{n}^{2}, x\right)$ is a strictly increasing function on $[0, \infty)$

\section{Theorem 1.7}

If $D_{t}\left(W_{n}^{2}, i\right)$ and $D_{t}\left(C_{n}^{2}, i\right)$ are the collection of total dominating sets of $W_{n}^{2}$ and $C_{n}^{2}$ respectively with cardinality I, where $i>\left\lceil\frac{n}{5}\right\rceil+1$ then $d_{t}\left(W_{n}^{2}, i\right)=n c_{i-1}+d_{t}\left(C_{n}^{2}, i\right)$

Proof:

$$
\text { We have } \begin{aligned}
\mathrm{d}_{\mathrm{t}}\left(\mathrm{W}_{\mathrm{n}}^{2}, \mathrm{i}\right) & =\mathrm{d}_{\mathrm{t}}^{0}\left(\mathrm{~W}_{\mathrm{n}}^{2}, \mathrm{i}\right)+\mathrm{d}_{\mathrm{t}}^{1}\left(\mathrm{~W}_{\mathrm{n}}^{2}, \mathrm{i}\right) \\
& =\mathrm{d}_{\mathrm{t}}^{0}\left(\mathrm{~W}_{\mathrm{n}}^{2}, \mathrm{i}\right)+\mathrm{d}_{\mathrm{t}}\left(\mathrm{C}_{\mathrm{n}}^{2}, \mathrm{i}\right)
\end{aligned}
$$

The number of total dominating sets of $\mathrm{W}_{\mathrm{n}}^{2}$ containing the vertex $\mathrm{v}_{0}$ with cardinality $\mathrm{i}$ is the number of ways to choose $\mathrm{i}-1$ vertices from the vertices $1,2,3, \ldots \ldots \ldots, n$. Therefore $d_{t}^{0}\left(W_{n}^{2}, i\right)=n c_{i-1}$. Therefore $d_{t}\left(W_{n}^{2}, i\right)=$ $\mathrm{nc}_{\mathrm{i}-1}+\mathrm{d}_{\mathrm{t}}\left(\mathrm{C}_{\mathrm{n}}^{2}, \mathrm{i}\right)$.

\section{Lemma 1.8}

$\mathrm{D}_{\mathrm{t}}\left(\mathrm{W}_{\mathrm{n}}^{2}, \mathrm{i}\right) \neq \varphi$, then we have

Let $\mathrm{W}_{\mathrm{n}}^{2}, \mathrm{n} \geq 3$ be the square of path with $\left|\mathrm{V}\left(\mathrm{W}_{\mathrm{n}}^{2}\right)\right|=\mathrm{n}+1$. Suppose that

(i) $\quad D_{t}\left(W_{n-2}^{2}, i-1\right)=\varphi$ and $D_{t}\left(W_{n-1}^{2}, i-1\right) \neq \varphi$ if and only if $i=n+1$.

(ii) $\quad \mathrm{D}_{\mathrm{t}}\left(\mathrm{W}_{\mathrm{n}-1}^{2}, \mathrm{i}-1\right) \neq \varphi, \mathrm{D}_{\mathrm{t}}\left(\mathrm{W}_{\mathrm{n}-2}^{2}, \mathrm{i}-1\right) \neq \varphi$ and $\mathrm{D}_{\mathrm{t}}\left(\mathrm{W}_{\mathrm{n}-3}^{2}, \mathrm{i}-1\right)=\varphi$ if only if $\mathrm{i}=\mathrm{n}$.

\section{Proof of (i)}

Suppose, $D_{t}\left(W_{n-2}^{2}, \mathrm{i}-1\right)=\varphi$ and $D_{t}\left(W_{n-1}^{2}, i-1\right) \neq \varphi$

$\Longrightarrow \mathrm{d}_{\mathrm{t}}\left(\mathrm{W}_{\mathrm{n}-2}^{2}, \mathrm{i}-1\right)=0$ and $\mathrm{d}_{\mathrm{t}}\left(\mathrm{W}_{\mathrm{n}-1}^{2}, \mathrm{i}-1\right) \neq 0$

$\Rightarrow \mathrm{i}-1<\mathrm{Y}_{\mathrm{t}}\left(\mathrm{W}_{\mathrm{n}}^{2}\right) \quad$ or $\mathrm{i}-1>\mathrm{n}-1$ and $\mathrm{Y}_{\mathrm{t}}\left(\mathrm{W}_{\mathrm{n}}^{2}\right) \leq \mathrm{i}-1 \leq \mathrm{n}$

If $\mathrm{i}-1<\mathrm{Y}_{\mathrm{t}}\left(\mathrm{W}_{\mathrm{n}}^{2}\right) \quad \Longrightarrow \mathrm{i}-1<\mathrm{i}<\mathrm{Y}_{\mathrm{t}}\left(\mathrm{W}_{\mathrm{n}}^{2}\right) \quad \Rightarrow \mathrm{i}<\mathrm{Y}_{\mathrm{t}}\left(\mathrm{W}_{\mathrm{n}}^{2}\right)$

$\Rightarrow d_{t}\left(W_{n}^{2}, i\right)=0$ which is a contradiction, since $d_{t}\left(W_{n}^{2}, i\right) \neq 0$, so $\mathrm{i}-1<Y_{t}\left(W_{n}^{2}\right)$ is not possible. Therefore $\mathrm{i}-1>$ n-1.

$\Rightarrow \mathrm{i}>\mathrm{n} \Rightarrow \mathrm{i} \geq \mathrm{n}+1$

Also, we have $\mathrm{i}-1 \leq \mathrm{n}$

$$
\Rightarrow \mathrm{i} \leq \mathrm{n}+1
$$

From (1) and (2) we get $\mathrm{i}=\mathrm{n}+1$. 
Conversely, if $\mathrm{i}=\mathrm{n}+1$, then

$\mathrm{D}_{\mathrm{t}}\left(\mathrm{W}_{\mathrm{n}-2}^{2}, \mathrm{i}-1\right)=\mathrm{D}_{\mathrm{t}}\left(\mathrm{W}_{\mathrm{n}-2}^{2}, \mathrm{n}\right)=\varphi$ and

$\mathrm{D}_{\mathrm{t}}\left(\mathrm{W}_{\mathrm{n}-1}^{2}, \mathrm{i}-1\right)=\mathrm{D}_{\mathrm{t}}\left(\mathrm{W}_{\mathrm{n}-1}^{2}, \mathrm{n}\right) \neq \varphi$.

Proof of (ii)

Suppose,

$\mathrm{D}_{\mathrm{t}}\left(\mathrm{W}_{\mathrm{n}-1}^{2}, \mathrm{i}-1\right) \neq \varphi, \mathrm{D}_{\mathrm{t}}\left(\mathrm{W}_{\mathrm{n}-2}^{2}, \mathrm{i}-1\right) \neq \varphi$

Then $\mathrm{d}_{\mathrm{t}}\left(\mathrm{W}_{\mathrm{n}-1}^{2}, \mathrm{i}-1\right) \neq 0, \mathrm{~d}_{\mathrm{t}}\left(\mathrm{W}_{\mathrm{n}-2}^{2}, \mathrm{i}-1\right) \neq 0$

$\Rightarrow \mathrm{Y}_{\mathrm{t}}\left(\mathrm{W}_{\mathrm{n}}^{2}\right) \leq \mathrm{i}-1 \leq \mathrm{n}$ and $\mathrm{Y}_{\mathrm{t}}\left(\mathrm{W}_{\mathrm{n}}^{2}\right) \leq \mathrm{i}-1 \leq \mathrm{n}-1$

$\Rightarrow \mathrm{i}-1 \leq \mathrm{n}-1$

$\Rightarrow \mathrm{i} \leq \mathrm{n}$

Also we have $\mathrm{D}_{\mathrm{t}}\left(\mathrm{W}_{\mathrm{n}-3}^{2}, \mathrm{i}-1\right)=\varphi$

Then $\mathrm{d}_{\mathrm{t}}\left(\mathrm{W}_{\mathrm{n}-3}^{2}, \mathrm{i}-1\right)=0$

$\Rightarrow \mathrm{i}-1<\mathrm{Y}_{\mathrm{t}}\left(\mathrm{W}_{\mathrm{n}}^{2}\right) \quad$ or $\mathrm{i}-1>\mathrm{n}-2$

If $\mathrm{i}-1<\mathrm{Y}_{\mathrm{t}}\left(\mathrm{W}_{\mathrm{n}}^{2}\right) \Rightarrow \mathrm{i}-1<\mathrm{i}<\mathrm{Y}_{\mathrm{t}}\left(\mathrm{W}_{\mathrm{n}}^{2}\right) \Rightarrow \mathrm{i}<\mathrm{Y}_{\mathrm{t}}\left(\mathrm{W}_{\mathrm{n}}^{2}\right)$

$\Longrightarrow \quad d_{t}\left(W_{n}^{2}, i-1\right)=0$ which is a contradiction, since $d_{t}\left(W_{n}^{2}, i\right) \neq 0$.

Therefore $\mathrm{i}-1<\mathrm{Y}_{\mathrm{t}}\left(\mathrm{W}_{\mathrm{n}}^{2}\right)$ is not possible, so $\mathrm{i}-1>\mathrm{n}-2$

$\Longrightarrow \quad \mathrm{i}>\mathrm{n}-1$

$\Longrightarrow \quad \mathrm{i} \geq \mathrm{n}$

From (1) and (2) we get $\mathrm{i}=\mathrm{n}$.

Conversely, if $\mathrm{i}=\mathrm{n}$, then

$\mathrm{D}_{\mathrm{t}}\left(\mathrm{W}_{\mathrm{n}-1}^{2}, \mathrm{i}-1\right)=\mathrm{D}_{\mathrm{t}}\left(\mathrm{W}_{\mathrm{n}-1}^{2}, \mathrm{n}-1\right) \neq \varphi$

$\mathrm{D}_{\mathrm{t}}\left(\mathrm{W}_{\mathrm{n}-2}^{2}, \mathrm{i}-1\right)=\mathrm{D}_{\mathrm{t}}\left(\mathrm{W}_{\mathrm{n}-2}^{2}, \mathrm{n}-1\right) \neq \varphi$ and

$D_{t}\left(W_{n-3}^{2}, i-1\right)=D_{t}\left(W_{n-3}^{2}, n-1\right)=\varphi$

Theorem 1.9

Let $\mathrm{W}_{\mathrm{n}}^{2}, \mathrm{n} \geq 3$ be the square of wheel with $\left|\mathrm{V}\left(\mathrm{W}_{\mathrm{n}}^{2}\right)\right|=\mathrm{n}+1$. Then the following properties hold for the coefficients of $\mathrm{D}_{\mathrm{t}}\left(\mathrm{W}_{\mathrm{n}}^{2}, \mathrm{x}\right)$ :

(i) For $\mathrm{n} \geq 2, \mathrm{~d}_{\mathrm{t}}\left(\mathrm{W}_{\mathrm{n}}^{2}, \mathrm{n}+1\right)=1$

(ii) For $\mathrm{n} \geq 2, \mathrm{~d}_{\mathrm{t}}\left(\mathrm{P}_{\mathrm{n}}^{2}, \mathrm{n}\right)=\mathrm{n}+1$

(iii) For $\mathrm{n} \geq 3, \mathrm{~d}_{\mathrm{t}}\left(\mathrm{W}_{\mathrm{n}}^{2}, \mathrm{n}-1\right)=(\mathrm{n}+1) \mathrm{c}_{2}$

(iv) For $\mathrm{n} \geq 5, \mathrm{~d}_{\mathrm{t}}\left(\mathrm{W}_{\mathrm{n}}^{2}, \mathrm{n}-2\right)=(\mathrm{n}+1) \mathrm{c}_{3}$

(v) For $\mathrm{n} \geq 5, \mathrm{~d}_{\mathrm{t}}\left(\mathrm{W}_{\mathrm{n}}^{2}, \mathrm{n}-3\right)=(\mathrm{n}+1) \mathrm{c}_{4}$

(vi) For $\mathrm{n} \geq 7, \mathrm{~d}_{\mathrm{t}}\left(\mathrm{W}_{\mathrm{n}}^{2}, \mathrm{n}-4\right)=(\mathrm{n}+1) \mathrm{c}_{5}-\mathrm{n}$

Proof of (i)

Since for any graph $\mathrm{G}$ with $\mathrm{n}+1$ vertices, $\mathrm{d}_{\mathrm{t}}(\mathrm{G}, \mathrm{n}+1)=1$, then

\section{Proof of (ii)}

$$
\mathrm{d}_{\mathrm{t}}\left(\mathrm{W}_{\mathrm{n}}^{2}, \mathrm{n}+1\right)=1 \text {. }
$$

To prove $\mathrm{d}_{\mathrm{t}}\left(\mathrm{W}_{\mathrm{n}}^{2}, \mathrm{n}\right)=\mathrm{n}+1$, for $\mathrm{n} \geq 2$.

We have, $\mathrm{d}_{\mathrm{t}}\left(\mathrm{W}_{\mathrm{n}}^{2}, \mathrm{i}\right)=\mathrm{nc}_{\mathrm{i}-1}+\mathrm{d}_{\mathrm{t}}\left(\mathrm{C}_{\mathrm{n}}^{2}, \mathrm{i}\right)$

$\mathrm{d}_{\mathrm{t}}\left(\mathrm{W}_{\mathrm{n}}^{2}, \mathrm{n}\right)=\mathrm{nc}_{\mathrm{n}-1}+\mathrm{d}_{\mathrm{t}}\left(\mathrm{C}_{\mathrm{n}}^{2}, \mathrm{n}\right)$

$$
=\mathrm{n}+1
$$

Therefore $\mathrm{d}_{\mathrm{t}}\left(\mathrm{W}_{\mathrm{n}}^{2}, \mathrm{n}\right)=\mathrm{n}+1$.

\section{Proof of (iii)}

To prove $\mathrm{d}_{\mathrm{t}}\left(\mathrm{W}_{\mathrm{n}}^{2}, \mathrm{n}-1\right)=(\mathrm{n}+1) \mathrm{c}_{2}$, for $\mathrm{n} \geq 3$

We have, $d_{t}\left(W_{n}^{2}, i\right)=n c_{i-1}+d_{t}\left(C_{n}^{2}, i\right)$

$$
\begin{aligned}
\mathrm{d}_{\mathrm{t}}\left(\mathrm{W}_{\mathrm{n}}^{2}, \mathrm{n}-1\right)= & \mathrm{nc} \mathrm{c}_{\mathrm{n}-2}+\mathrm{d}_{\mathrm{t}}\left(\mathrm{C}_{\mathrm{n}}^{2}, \mathrm{n}-1\right) \\
& =\frac{1}{2} \mathrm{n}(\mathrm{n}-1)+\mathrm{n} \\
& =\frac{1}{2} \mathrm{n}(\mathrm{n}-1+2) \\
& =\frac{1}{2} \mathrm{n}(\mathrm{n}+1) \\
& =(\mathrm{n}+1) \mathrm{c}_{2}
\end{aligned}
$$

Proof of (iv)

$$
=(n+1) c_{2}-2, \text { for } n \geq 5
$$

To prove $d_{t}\left(W_{n}^{2}, n-2\right)=(n+1) c_{3}$ for every $n \geq 5$

We have, $\mathrm{d}_{\mathrm{t}}\left(\mathrm{W}_{\mathrm{n}}^{2}, \mathrm{i}\right)=\mathrm{nc}_{\mathrm{i}-1}+\mathrm{d}_{\mathrm{t}}\left(\mathrm{C}_{\mathrm{n}}^{2}, \mathrm{i}\right)$

$$
\mathrm{d}_{\mathrm{t}}\left(\mathrm{W}_{\mathrm{n}}^{2}, \mathrm{n}-2\right)=\mathrm{nc}_{\mathrm{n}-3}+\mathrm{d}_{\mathrm{t}}\left(\mathrm{C}_{\mathrm{n}}^{2}, \mathrm{n}-2\right)
$$




$$
\begin{aligned}
& =\frac{1}{6}[n(n-1)(n-2)]-2(n-2)+\frac{1}{2} n(n-1) \\
& =\frac{1}{6} n(n-1)[n-2+3] \\
& =\frac{1}{6} n(n-1)(n+1) \\
& =\frac{1}{6}(n+1) n(n-1) \\
& =(n+1) c_{3}
\end{aligned}
$$

$d_{t}\left(W_{n}^{2}, n-2\right)=(n+1) c_{3}$ for every $n \geq 5$

\section{Proof of (v)}

To prove $d_{t}\left(W_{n}^{2}, n-3\right)=(n+1) c_{4}$, for $n \geq 5$.

We have, $d_{t}\left(W_{n}^{2}, i\right)=n c_{i-1}+d_{t}\left(C_{n}^{2}, i\right)$

$$
\begin{aligned}
\mathrm{d}_{\mathrm{t}}\left(\mathrm{W}_{\mathrm{n}}^{2}, \mathrm{n}-3\right)= & \mathrm{nc} \mathrm{c}_{\mathrm{n}-4}+\mathrm{d}_{\mathrm{t}}\left(\mathrm{C}_{\mathrm{n}}^{2}, \mathrm{n}-3\right) \\
= & \frac{1}{24}[\mathrm{n}(\mathrm{n}-1)(\mathrm{n}-2)(\mathrm{n}-3)]-2(\mathrm{n}-2)+\frac{1}{6} \mathrm{n}(\mathrm{n}-1)(\mathrm{n}-2) \\
& =\frac{1}{24} \mathrm{n}(\mathrm{n}-1)(\mathrm{n}-2)[\mathrm{n}-3+4] \\
& =\frac{1}{24} \mathrm{n}(\mathrm{n}-1)(\mathrm{n}-2)(\mathrm{n}+1) \\
& =\frac{1}{24}(\mathrm{n}+1) \mathrm{n}(\mathrm{n}-1)(\mathrm{n}-2) \\
& =(\mathrm{n}+1) \mathrm{c}_{4}
\end{aligned}
$$

$d_{t}\left(W_{n}^{2}, n-3\right)=(n+1) c_{4}$, for $n \geq 5$

\section{Proof of (vi)}

To prove $d_{t}\left(W_{n}^{2}, n-4\right)=(n+1) c_{5}-n$, for $n \geq 7$.

We have, $d_{t}\left(W_{n}^{2}, i\right)=n c_{i-1}+d_{t}\left(C_{n}^{2}, i\right)$

$$
\begin{aligned}
d_{t}\left(W_{n}^{2}, n-4\right)= & n c_{n-5}+d_{t}\left(C_{n}^{2}, n-4\right) \\
= & \frac{1}{120}[n(n-1)(n-2)(n-3)(n-4)]-2(n-2)+\frac{1}{24} n(n-1)(n-2)(n-3)-n \\
= & \frac{1}{120} n(n-1)(n-2)(n-3)[n-4+5]-n \\
= & \frac{1}{120} n(n-1)(n-2)(n-3)(n+1)-n \\
= & \frac{1}{120}(n+1) n(n-1)(n-2)(n-3)-n \\
& =(n+1) c_{5}-n
\end{aligned}
$$

$d_{t}\left(W_{n}^{2}, n-4\right)=(n+1) c_{5}-n$, for $n \geq 7$.

Using theorem 1.7 and theorem 1.9 , we obtain $d_{t}\left(W_{n}^{2}, i\right)$ for $1 \leq \mathrm{i} \leq 11$ as shown in Table 1.1

Table1.1 $d_{t}\left(W_{n}^{2}, i\right)$, the number of total dominating sets of $W_{n}^{2}$ with cardinality $i$

\begin{tabular}{|l|l|l|l|l|l|l|l|l|l|l|}
\hline $\mathrm{i}$ & 2 & 3 & 4 & 5 & 6 & 7 & 8 & 9 & 10 & 11 \\
\hline $\mathrm{n}$ & & & & & & & & & & \\
\hline 3 & 6 & 4 & 1 & & & & & & & \\
\hline 4 & 10 & 10 & 5 & 1 & & & & & & \\
\hline 5 & 15 & 20 & 15 & 6 & 1 & & & & & \\
\hline 6 & 18 & 35 & 35 & 21 & 7 & 1 & & & & \\
\hline 7 & 14 & 49 & 70 & 56 & 28 & 8 & 1 & & & \\
\hline 8 & 8 & 52 & 118 & 126 & 84 & 36 & 9 & 1 & & \\
\hline 9 & 9 & 45 & 165 & 243 & 210 & 120 & 45 & 10 & 1 & \\
\hline 10 & 10 & 55 & 201 & 403 & 452 & 330 & 165 & 55 & 11 & 1 \\
\hline
\end{tabular}

\section{Theorem 1.10}

If $\mathrm{D}_{\mathrm{t}}\left(\mathrm{W}_{\mathrm{n}}^{2}, \mathrm{x}\right)$ is the total dominating polynomial of square of wheel $\mathrm{W}_{\mathrm{n}}^{2}$, then $\mathrm{D}_{\mathrm{t}}\left(\mathrm{W}_{\mathrm{n}}^{2}, \mathrm{x}\right)=\mathrm{x}\left[(1+\mathrm{x})^{\mathrm{n}}-1\right]+$ $\mathrm{D}_{\mathrm{t}}\left(\mathrm{W}_{\mathrm{n}}^{2}, \mathrm{x}\right)$

Proof:

We have, $\mathrm{D}_{\mathrm{t}}\left(\mathrm{W}_{\mathrm{n}}^{2}, \mathrm{x}\right)=\sum_{\mathrm{i}=2}^{\mathrm{n}+1} \mathrm{~d}_{\mathrm{t}}\left(\mathrm{W}_{\mathrm{n}}^{2}, \mathrm{i}\right) \mathrm{x}^{\mathrm{i}}$

$$
\begin{aligned}
& =\sum_{i=2}^{Y_{t}\left(C_{n}^{2}\right)-1} d_{t}\left(W_{n}^{2}, i\right) x^{i}+\sum_{i=Y_{t}\left(C_{n}^{2}\right)}^{n+1} d_{t}\left(W_{n}^{2}, i\right) x^{i} \\
& =\sum_{i=2}^{Y_{t}\left(C_{n}^{2}\right)-1} d_{t}^{0}\left(W_{n}^{2}, i\right) x^{i}+\sum_{i=Y_{t}}^{n+1}\left(C_{n}^{2}\right)\left\{n c_{i-1}+d_{t}\left(C_{n}^{2}, i\right)\right\} x^{i} \\
& =\sum_{i=2}^{Y_{t}\left(C_{n}^{2}\right)-1} n_{i-1} x^{i}+\sum_{i=Y_{t}}^{n+1}\left(C_{n}^{2}\right) n c_{i-1} x^{i}+\sum_{i=Y_{t}}^{n+1}\left(C_{n}^{2}\right) d_{t}\left(C_{n}^{2}, i\right) x^{i} \\
& =\sum_{i=2}^{Y_{t}\left(C_{n}^{2}\right)-1} n_{i-1} x^{i}+\sum_{i=Y_{t}}^{n+1}\left(C_{n}^{2}\right) d_{t}\left(C_{n}^{2}, i\right) x^{i} \\
= & x \sum_{i=2}^{n+1} n c_{i-1} x^{i-1}+D_{t}\left(C_{n}^{2}, x\right) \\
D_{t}\left(W_{n}^{2}, x\right)= & x\left[(1+x)^{n}-1\right]+D_{t}\left(C_{n}^{2}, x\right)
\end{aligned}
$$

